
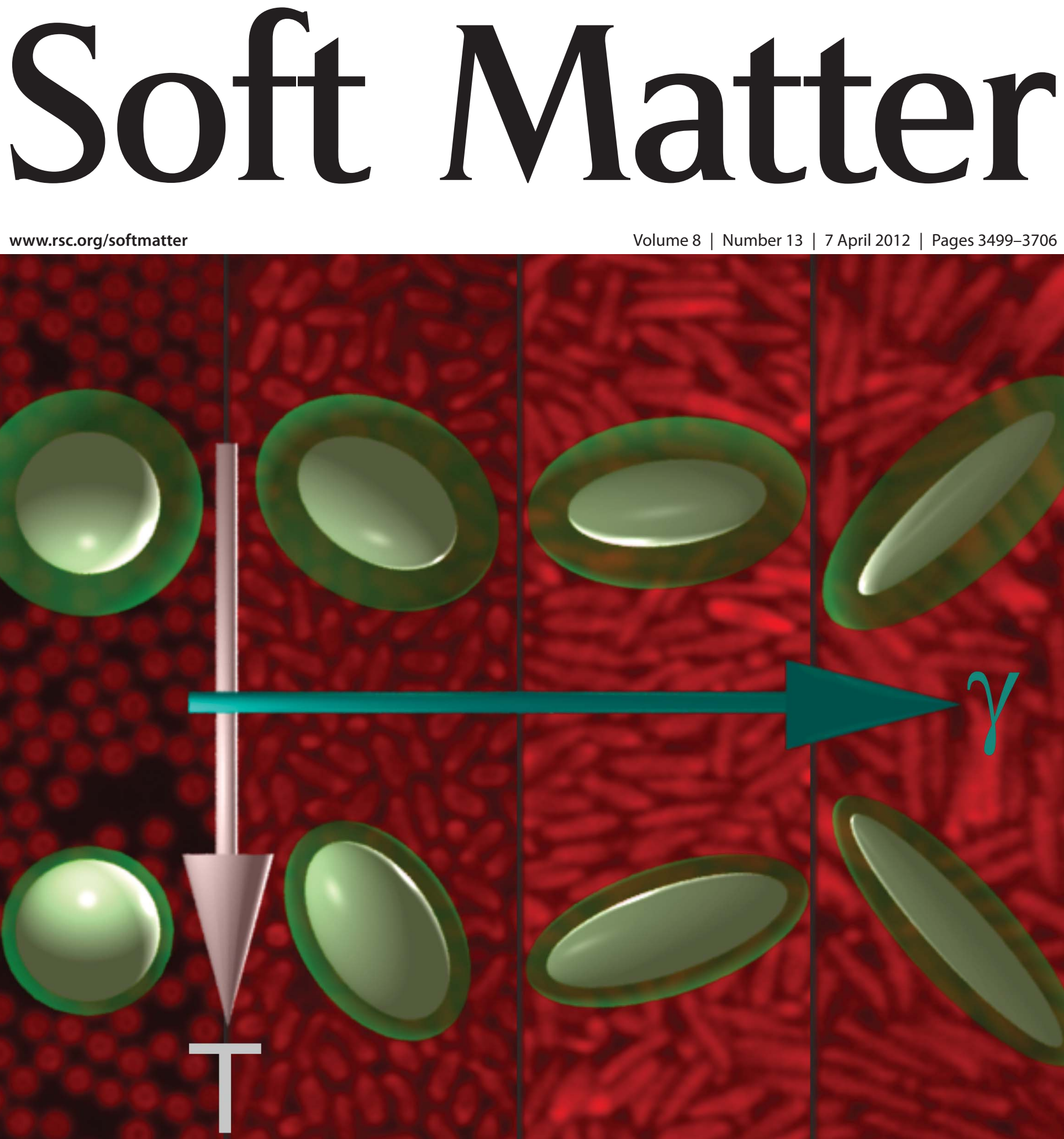


\title{
Preparation and characterization of ellipsoidal-shaped thermosensitive microgel colloids with tailored aspect ratios
}

\author{
Jérôme J. Crassous, ${ }^{* a e}$ Hervé Dietsch, ${ }^{* a}$ Patrick Pfleiderer, ${ }^{b}$ Vikash Malik, ${ }^{a}$ Ana Diaz, ${ }^{c}$ \\ Liliane Ackermann Hirshi, ${ }^{a}$ Markus Drechsler ${ }^{d}$ and Peter Schurtenberger ${ }^{e}$
}

Received 28th November 2011, Accepted 24th January 2012

DOI: 10.1039/c2sm07257b

Prolate model colloids with defined properties can be obtained by the stretching of spherical polymeric particles, which is well-known for polystyrene and poly(methyl methacrylate) latices. The present study aims to extend this approach to functional core-shell particles in order to achieve a new class of anisotropic colloidal materials where both the aspect ratio and the effective volume fraction can be controlled by temperature. We describe the synthesis and characterization of these functional anisotropic core-shell particles consisting of a polystyrene (PS) core onto which a crosslinked thermoresponsive microgel shell of poly( $N$-isopropylmethacrylamide) (PNIPMAm) was grafted. Embedded into a film of polyvinylalcohol (PVA), the spherical composite microgels are heated above the glass transition temperature $\left(T_{\mathrm{g}}\right)$ of the polystyrene core and then stretched with different deformations $\gamma$. During the stretching, the particles adopt an elongated shape, that they retain after cooling to room temperature as confirmed by small-angle X-ray scattering performed on the films. Anisotropic composite microgels with aspect ratios ranging from 2.2 to 6.5 are recovered after dissolution of the PVA and purification, and are fully characterized by diverse methods such as transmission electron microscopy, confocal microscopy and light scattering. The temperature sensitivity of these anisotropic composite microgels is maintained as confirmed by dynamic light scattering and cryogenic electron microscopy performed below and above the volume phase transition of the shell.

\section{Introduction}

Designing various anisotropic colloidal particles opens new possibilities to explore complex phase diagrams. ${ }^{1-5}$ Complexity resides in their shape anisotropy, which is reflected by their specific dynamics ${ }^{6-8}$ and structural organization. At higher volume fraction, fascinating dynamics and complex phase diagrams have been predicted depending upon the aspect ratio and the interparticle interactions. ${ }^{4,5,9-13}$ Thus, interest resides in the study of liquid crystalline phases at the colloidal level ${ }^{14}$ with a potential to extend it to complex biological systems with an anisotropic architecture and/or interaction potential such as blood cells, viruses, proteins or bacteria.

Among anisotropic particles, ellipsoidal particles and particularly their phase diagram at high volume fractions have been

${ }^{a}$ Adolphe Merkle Institute, University Fribourg, Route de l'Ancienne Papeterie, PO Box 209, 1723 Marly 1, Switzerland. E-mail: jerome. crassous@fkem1.lu.se; herve.dietsch@unifr.ch

${ }^{b}$ Department of Chemical Engineering, Katholieke Universiteit Leuven, 3001 Heverlee, Belgium

${ }^{c}$ Paul Scherrer Institute, 5232 Villigen, PSI, Switzerland

${ }^{d}$ Makromolekular Chemistry II, Bayreuth University, 95440 Bayreuth, Germany

${ }^{e}$ Physical Chemistry, Center for Chemistry and Chemical Engineering, Lund University, 22100 Lund, Sweden much less studied experimentally. This is due to the challenges in synthesizing monodisperse model anisotropic colloids combined with the required development of appropriate methods of characterization. Synthetic colloidal prolates are not only appealing for fundamental physics but also for nanotechnological applications. As an example, their reorganization in ordered structures can be employed for designing new photonic materials. ${ }^{15,16}$ Moreover their incorporation into a polymeric elastomer was found to mechanically reinforce the composite and to provide light or X-ray polarization upon material deformation. ${ }^{17}$ Ellipsoidal particles can be made with a variety of methods. One such method relies on the oriented crystalline growth of an inorganic material during the synthesis ${ }^{18,19}$ such as for example hematite spindles ${ }^{18,20,21}$ with canted-antiferromagnetic properties, ${ }^{22}$ where size and shape can be adjusted by chemical triggers. These particles can be further coated with silica of different thicknesses leading to the control of the final aspect ratio. ${ }^{\mathbf{8 , 2 1 , 2 3 - 2 5}}$ Moreover, this coating enables different surface chemistry using silane coupling, which allows the integration of the particles in microgels, ${ }^{26}$ or in a bulk polymeric matrix. ${ }^{17,27}$

Another method of achieving ellipsoidal particles involves amorphous polymeric latices, which can be mechanically stretched following the method originally developed by Keville et al. ${ }^{28}$ for poly(methyl methacrylate) particles and then extended 
to polystyrene particles by Ho et al. ${ }^{29}$ The particles are embedded into a polymeric film, which is maintained above the glass transition temperature of the particles and subsequently stretched at different uniaxial deformations $\gamma$, where the particles adopt an anisotropic shape that they retain after cooling the film. The particles can then be recovered after dissolution of the film and subsequent purification. Since a uniaxial deformation results in well-defined prolates, ${ }^{28-31}$ a large variety of particle designs can be achieved via a multidirectional deformation as shown by Champion et al. ${ }^{32}$ This method allows a fine tuning of the shape and aspect ratio as it basically depends on the applied deformation. The regained interest in these polymeric prolates was demonstrated in recent studies in various fields dealing, for example, with their dynamics in bulk ${ }^{6,7}$ and at an interface, ${ }^{33}$ their use as pickering agents ${ }^{34}$ and their organization in an electric field ${ }^{35,36}$ or in the dense state after application of a centrifugal field. ${ }^{30,37}$ In comparison to inorganic materials, highly monodisperse polystyrene lattices can easily be synthesized by emulsion polymerization in water in a large quantity. Moreover their lower refractive index and density make them more suitable for many studies. They are also easy to functionalize either with a dense polyelectrolyte brush or with a microgel shell, ${ }^{38,39}$ which provides an additional responsivity to $\mathrm{pH}$, salt, or temperature.

As an example spherical PS/PNIPAm core-shell particles have been intensively used as model systems for the dynamics of colloids in the vicinity of the glass transition profiting from the fact that their size, and the resulting effective volume fraction can be efficiently adjusted as a function of the temperature. ${ }^{40,41}$ The structure of these particles and the volume phase transition (VPT) of the PNIPAm shell have been characterized by diverse methods such as SANS and SAXS,${ }^{42}$ or cryoTEM. ${ }^{39,43,44}$ At high ionic strength, it was also recently demonstrated that the interactions can be tuned from repulsive to attractive with the temperature leading to the reversible aggregation of this system above the volume phase transition temperature $T_{\mathrm{VPT}}{ }^{40,45}$

This contribution aims to extend the uniaxial stretching method to novel themosensitive core-shell polystyrene/poly( $N$-isopropylmethacrylamide) (PS/PNIPMAm) microgels, which present many similarities to spherical PS/PNIPAm composite microgels. In comparison to former work using anisotropic

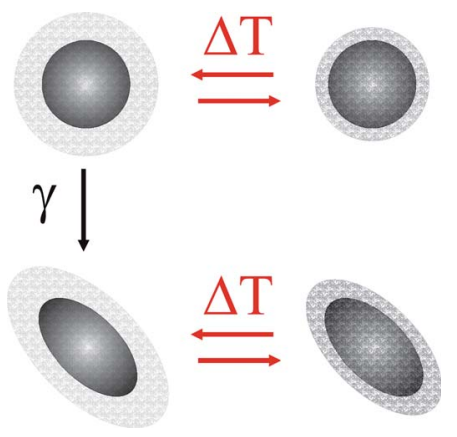

Fig. 1 Schematic representation of the core-shell particles. The system consists of PS/PNIPMAm core-shell particles. Dispersed into water, the PNIPMAm shell swells and deswells as a function of the temperature. The original spherical colloidal particles can be elongated into ellipsoidal ones of different aspect ratios maintaining the thermosensitivity of the shell. particles as a core for the synthesis of core-shell microgels, ${ }^{26}$ this approach is expected to lead to anisotropic particles with higher aspect ratios while keeping the thermosensitivity of the shell as shown in Fig. 1. We focus on the complete characterization of these new systems by means of diverse methods. The conformation of the particles within the films were investigated by SAXS. The dispersed particles are imaged either in the dried state by transmission electron microscopy (TEM) or in solution via confocal and cryogenic electron (CryoTEM) microscopy. Their response to temperature in suspension was further characterized using static and dynamic light scattering.

\section{Experimental}

\subsection{Chemicals}

$N$-Isopropylmethacrylamide (NIPMAM; Aldrich), $N, N^{\prime}$-methylenebisacrylamide (BIS; Fluka) monomers, sodium dodecyl sulfate (SDS; Fluka), and potassium peroxodisulfate (KPS; Fluka), polyvinyl alcohol (PVA; 89-98 kDa; 99\%; Aldrich) were used as received. Styrene monomer (BASF) was purified on a $\mathrm{Al}_{2} \mathrm{O}_{3}$ column prior to use. Water was purified using reverse osmosis (MilliRO; Millipore) and ion exchange (MilliQ; Millipore).

\subsection{Latex core synthesis}

The core-shell particles were synthesized in a two-step reaction. ${ }^{46}$ In the first step, core particles were obtained by emulsion polymerization and used in the second step as seeds for radical polymerization of the respective crosslinked shells. Emulsion polymerization was performed in a one liter three necked round bottom flask equipped with a stirrer, a reflux condenser, and a thermometer. $0.014 \mathrm{~g}$ SDS and $2.625 \mathrm{~g}$ NIPMAm were dissolved in $50 \mathrm{~mL}$ pure water under continuous magnetic stirring; the obtained solution was degassed with nitrogen for $20 \mathrm{~min}$. After the addition of $48.3 \mathrm{~g}$ of styrene, the mixture was heated to $80{ }^{\circ} \mathrm{C}$ keeping a nitrogen atmosphere. $90 \mathrm{mg}$ of the KPS initiator were dissolved in parallel into $9.2 \mathrm{~mL}$ of water. This initiator solution was slowly added while the mixture was stirred at 300 $\mathrm{rpm}$. After $8 \mathrm{~h}$ the latex solution was cooled to room temperature and filtered through glass wool to remove traces of coagulum. Purification was performed by dialysis of the latex solution against pure MilliQ water solution for three weeks (Medicell, 12000-14000 Da). At the end of the process a $5.1 \mathrm{wt} \%$ core stock solution was obtained. We notice that without the addition of a small amount of NIPMAm no core-shell can be obtained in the second reaction step. This addition ensures that the surface of the core becomes hydrophobic at high temperatures, which enables the adsorption of PNIPMAm to the core during the core-shell synthesis.

\subsection{Latex core-shell synthesis}

The seeded emulsion polymerization for the core-shell system was carried out using $32 \mathrm{~g}$ of our stock core solution. $1.61 \mathrm{~g}$ of NIPMAm and $0.1 \mathrm{~g}$ of BIS were dissolved into $67 \mathrm{~g}$ of water and added to the core solution. The solution was then heated to $80{ }^{\circ} \mathrm{C}$. One hour later, the reaction was started by the slow addition of $50 \mathrm{mg}$ KPS (dissolved in $5 \mathrm{~mL}$ of water) and the 
entire mixture was stirred at $300 \mathrm{rpm}$ for $8 \mathrm{~h}$ at $80{ }^{\circ} \mathrm{C}$. After cooling to room temperature, the core-shell latex solution was filtered through glass wool and purified by dialysis against MilliQ water for two weeks. After dialysis the core-shell stock solution concentration was determined to be $3 \mathrm{wt} \%$.

\subsection{Mechanical deformation method}

The anisotropic particles were obtained based on the mechanical stretching method for PS lattices developed by Ho et al. ${ }^{29}$ Fig. 2 presents a summary of the full method adapted for our core-shell system. In a first step, hybrid PVA polymeric films containing either the core or the core-shell particles were prepared. In a typical example, $5 \mathrm{~mL}$ of a $2.3 \mathrm{wt} \%$ latex solution was added to $22 \mathrm{~mL}$ of a $7 \mathrm{wt} \%$ aqueous PVA solution. After homogenization and successive degassing of the solution, teflon molds were filled with this solution. After drying in the oven at $80^{\circ} \mathrm{C}$ for 3 days in a dessicator, PVA films containing about $9.7 \mathrm{w} / \mathrm{w}$ core-shell particles were recovered. Then the films were held between two clamps in a custom-made uniaxial deformation set-up. The setup is heated in an oven for approximately $15 \mathrm{~min}$ at $180{ }^{\circ} \mathrm{C}$, i.e. far above the glass transition temperature $T_{\mathrm{g}}$ of the polystyrene core, considering that the equilibrium $T_{\mathrm{g}}$ for polystyrene of a molecular weight higher than the critical value of about $100 \mathrm{~kg}$ $\mathrm{mol}^{-1}$ is about $105^{\circ} \mathrm{C} .{ }^{47}$ The different films were then stretched at different draw ratios, cooled to room temperature and sorted following their local deformations $\gamma$, which is accessible via a grid sketched on the film surface. Here we defined $\gamma=\Delta l / l_{0}$ where $\Delta l$ and $l_{0}$ respectively correspond to the difference of length before and after stretching and to the original size of the film.

\subsection{Characterization methods}

Small angle X-ray scattering (SAXS). Small angle X-ray scattering (SAXS) was performed on polymeric films stretched at different deformations at the cSAXS beamline at $11.2 \mathrm{keV}$ (corresponding to a wavelength of $\lambda=0.111 \mathrm{~nm}$ ) at the Swiss Light Source (Paul Scherrer Institute, Villigen, Switzerland). The stretch direction of the sample was tilted at an angle of about $45^{\circ}$ to avoid contributions of slit scattering along the deformation axis. The resulting $2 D$ patterns were first treated following the standard procedure (background subtraction and normalization) and then azimuthally averaged, either using a complete integration over the full range $0-2 \pi$, or using azimuthal sector average (using a sectoral angle of $\Delta \phi=\pi / 36$ ) only along two principle axis parallel and perpendicular to the stretch direction.

Transmission electron microscopy (TEM and cryo-TEM). Samples for conventional TEM were prepared by dropcasting a 1 $\mathrm{wt} \%$ solution on a 300 mesh carbon-coated copper grid placed on a paper filter at room temperature. The micrographs were taken with a TEM-CM100 (Philips) operating at an acceleration voltage of $80 \mathrm{keV}$. The cryogenic electron microscopy experiments were performed on dilute samples (about $0.2 \mathrm{wt} \%$ ) kept at a given temperature $\left(23{ }^{\circ} \mathrm{C}\right.$ or $\left.55^{\circ} \mathrm{C}\right)$ before vitrification. A few microlitres of diluted solutions were placed on a bare copper TEM grid (Plano, 600 mesh), and the excess liquid was removed with filter paper. This sample was cryo-fixed by rapid immersion into liquid ethane cooled to between $-170{ }^{\circ} \mathrm{C}$ and $-180{ }^{\circ} \mathrm{C}$ in a cryo-box (Carl Zeiss NTS GmbH). For the measurements above the volume phase transition, the TEM copper grid was placed in a controlled environment vitrification system (CEVS). The chamber was kept at $55{ }^{\circ} \mathrm{C}$ and was saturated with water vapor to prevent evaporation from the specimens. The vitrified specimen was inserted into a cryo-transfer holder (CT3500, Gatan, Munich, Germany) and transferred to a Zeiss EM922 EFTEM (Zeiss NTS GmbH, Oberkochen, Germany). Examination was carried out at temperatures around $90 \mathrm{~K}$. The TEM was operated at an acceleration voltage of $200 \mathrm{kV}$. Zero-loss filtered images were taken under reduced dose conditions. All images were recorded digitally by a bottom-mounted CCD camera system (UltraScan 1000, Gatan) and processed with a digital imaging processing system (Digital Micrograph 3.9 for GMS 1.4, Gatan).

Confocal microscopy. The confocal micrographs were monitored on a Leica SP5 confocal laser-scanning microscope (CLSM) operated in the inverted mode (D6000I). An

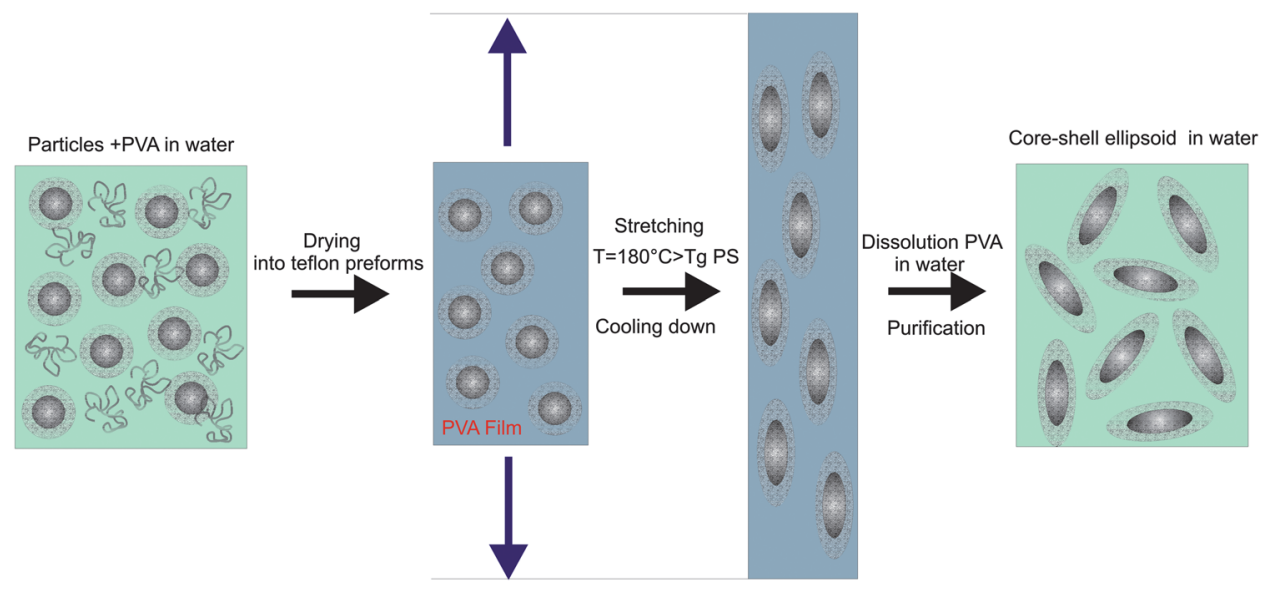

Fig. 2 Schematic representation of the ellipsoid preparation. Polyvinyl alcohol (PVA) is solubilized in water in the presence of the core-shell particles in the first step. The solution is then dried in teflon molds and forms PVA films with dispersed core-shell particles. The films are recovered and stretched in an oven at $180^{\circ} \mathrm{C}$ (far above the $T_{\mathrm{g}}$ of the polystyrene) and subsequently cooled to room temperature. The particles are finally recovered by dissolution of the films in hot water and repeated centrifugation. 
environmental system was employed to ensure a constant temperature set at $20{ }^{\circ} \mathrm{C}$ during all the examinations. The particles were non-covalently dyed after addition of water soluble Rhodamine B $\left(5 \times 10^{-3} \mathrm{~g} \mathrm{~L}^{-1}\right.$ in solution $)$ and prepared on a glass slide with a spacer of about $100 \mu \mathrm{m}$.

Static and dynamic light scattering (SLS and DLS). Static light scattering (SLS) and dynamic light scattering (DLS) were carried out using a light scattering goniometer instrument from LS Instruments equipped with a $\mathrm{He}-\mathrm{Ne} \operatorname{laser}(\lambda=632.8 \mathrm{~nm})$. The temperature was controlled with an accuracy of $0.1{ }^{\circ} \mathrm{C}$. The samples were highly diluted to $0.01 \mathrm{wt} \%$ preventing interaction effects and multiple scattering. The SLS measurements were performed from 20 to $144^{\circ}$, with an increment of $2^{\circ}$ and the DLS measurements at scattering angles of 45, 60 and $75^{\circ}$ for temperatures between $20^{\circ} \mathrm{C}$ and $50^{\circ} \mathrm{C}$.

\section{Results and discussion}

The synthesis of the core-shell particles proceeded in two steps. In the first step, a polystyrene core was synthesized by conventional emulsion polymerization in the presence of $5 \mathrm{wt} \%$ NIPMAm. The TEM analysis confirmed that the particles were practically monodisperse and well-defined. An average radius of $267 \mathrm{~nm}$ and a polydispersity of $7.5 \%$ were derived from the analysis made on 300 particles, in good agreement with the average hydrodynamic radius $R_{\mathrm{H}}$ of $254 \mathrm{~nm}$ determined between $20^{\circ} \mathrm{C}$ and $50^{\circ} \mathrm{C}$. As expected, the radius of the core particles as observed by DLS had no dependence on the temperature. It should be noted that the core particles bear a small number of charges on their surface originating from the remaining SDS and initiator fragments.
These charges insure the colloidal stability even at high temperatures. This point is of particular importance for the second step in which the thermosensitive shell was polymerized at $80{ }^{\circ} \mathrm{C}$ onto these core particles by a seeded emulsion polymerization. The original core-shell particles were imaged via TEM as shown in Fig. 6. The particles are monodisperse with an average radius determined from statistics of 100 particles to be $331 \mathrm{~nm}$ and a polydispersity of $5.6 \%$ in good agreement with the hydrodynamic radius of $349 \mathrm{~nm}$ determined above the VPT at $50{ }^{\circ} \mathrm{C}$ in the collapsed state. The $93 \mathrm{~nm}$ difference between the radii of the core and core-shell particles established from the TEM analysis clearly confirmed the presence of a dense PNIPAm shell at the surface of the particles. Assuming a density of $1.055 \mathrm{~g} \mathrm{~cm}^{-3}$ for the polystyrene core and the density of $1.129 \mathrm{~g} \mathrm{~cm}^{-3}$ for the PNIPMAm shell (measured in solution at $55^{\circ} \mathrm{C}^{49}$ ), the mass ratio core/shell could be approximated to be 1.03 . Compared to the unit mass ratio of the reactants prior synthesis, this analysis confirms the high degree of conversion and the efficiency of the polymerization. The conformation of the particles in the films was investigated via small -angle X-ray scattering (SAXS). The different scattering patterns obtained as function of $\gamma$ for both core and core-shell particles are reported in Fig. 3. The scattering intensity $I(q)$ measured for homogeneous prolate and oblate spheroids may be rendered as the product of $I_{0}(q)$, the scattering intensity of an isolated particle, and $S(q)$, the structure factor that takes into account the mutual interaction of the particles:

$$
I(q)=(N / V) I_{0}(q) S(q)
$$

where $N / V$ denotes the number density of the scattering objects. A previous discussion of $S(q)$ for systems of spherical particles
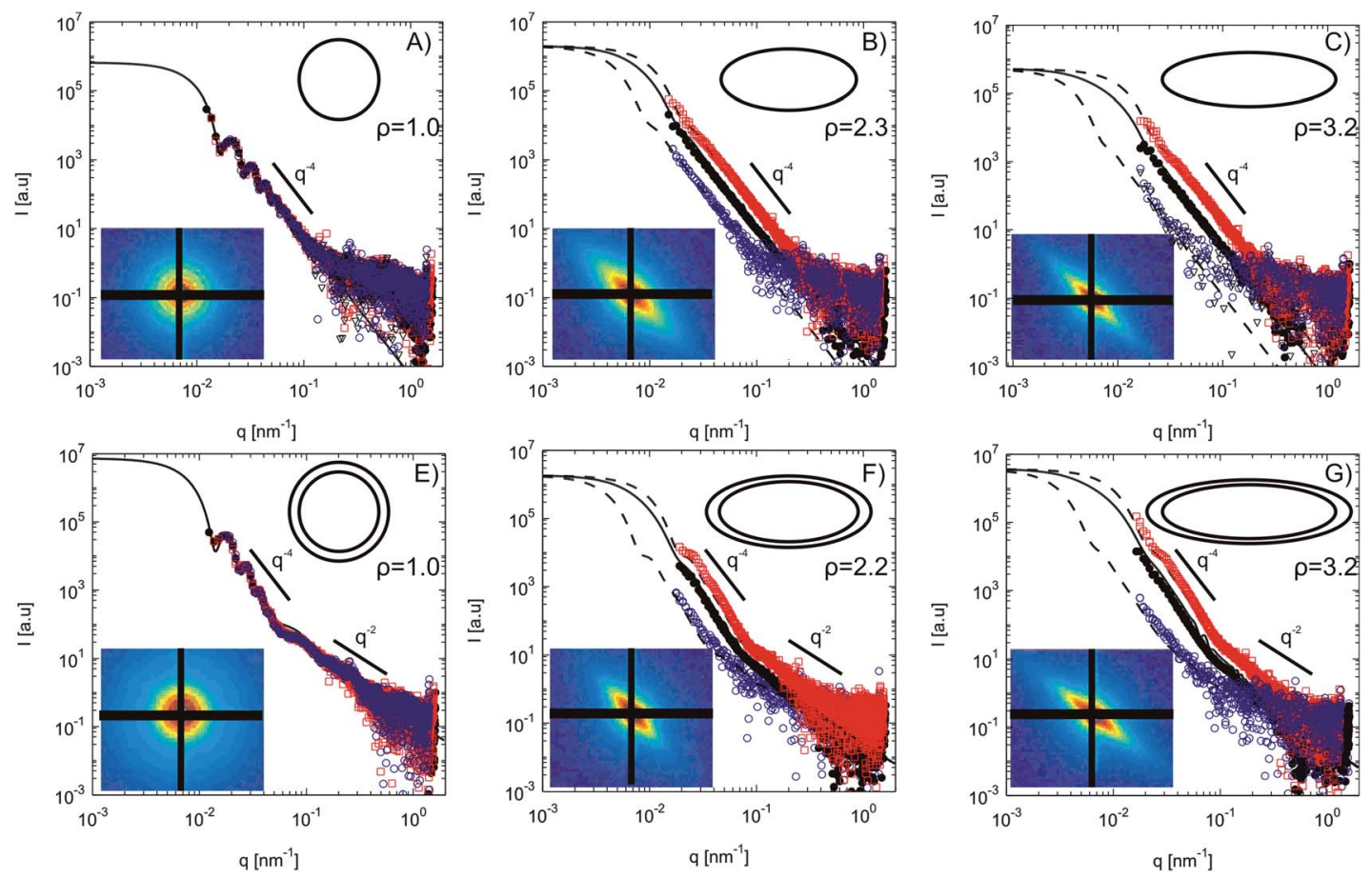

Fig. 3 SAXS investigation of the PVA films containing the PS core particles (A,B,C) and PS/PNIPMAm core-shell particles (E,F,G) elongated with different deformations $\gamma(0 \%$ : A,E; 50\%: B,F; 125\%: C,G) with their corresponding diffraction patterns. The scattering intensities following the short azimuthal (hollow squares) and long azimuthal (hollow circles), as well as the rotational averaged intensity (full circles) has been computed as function of the scattering vector $q$. The fit refers to the schematic models displayed on each graph with their corresponding aspect ratio $\rho$. 
has demonstrated that the influence of the structure factor is restricted to the region of smallest $q$ values when the concentration of the particles is small for well dispersed particles. ${ }^{42}$ Its influence on the measured scattering intensity can therefore be disregarded in the present analysis. Hence, $S(q)=1$ will be assumed in the following. The scattering intensity of one single particle can be decomposed in principle into three terms: ${ }^{42,46,50,51}$

$$
I_{0}(q)=I_{\text {part }}(q)+I_{\text {fluc }, \mathrm{PS}}(q)+I_{\text {fluc,shell }}(q)
$$

$I_{\text {part }}(q)$ is the part of $I_{0}(q)$ due to the core-shell structure of the particles (i.e., the scattering intensity caused by the composite particles having a homogeneous core and shell). ${ }^{42,46}$ The core, the shell and the surrounding medium are characterized by the scattering length densities, $\eta_{\mathrm{c}}, \eta_{\mathrm{sh}}$ and $\eta_{\mathrm{m}}$, respectively. $I_{\text {fluc,PS }}(q)$ and $I_{\text {fluc,shell }}(q)$ refer to the thermal fluctuation of the PS core and the PNIPMAm shell respectively. The shell, however, does not consist of a solid material but of a polymeric network that exhibits static inhomogeneities and thermal fluctuations, for this reason we neglected the contribution of the fluctuation of the PS core and we only take into account $I_{\text {fluc,shell }}(q)$. The scattering amplitude $F(q, \mu)$ of a core-shell prolate spheroid with semimajor core and core-shell axis $a_{\mathrm{c}}, a_{\mathrm{sh}}$ and semi-minor core and core-shell axis $b_{\mathrm{c}}, b_{\mathrm{sh}}$ (see Fig. 4) is given by ${ }^{52,53}$

$$
\begin{gathered}
F(q, \mu)=\left(\eta_{\mathrm{c}}-\eta_{\mathrm{sh}}\right) V_{\mathrm{c}}\left[\frac{3 j_{1}\left(u_{\mathrm{c}}\right)}{u_{\mathrm{c}}}\right]+\left(\eta_{\mathrm{sh}}-\eta_{\mathrm{m}}\right) V_{\mathrm{s}}\left[\frac{3 j_{1}\left(u_{\mathrm{sh}}\right)}{u_{\mathrm{sh}}}\right] \\
j_{1}(u)=(\sin (u)-u \cos (u)) / u^{2} \\
u_{\mathrm{c}}=q \sqrt{a_{\mathrm{c}}^{2} \mu^{2}+b_{\mathrm{c}}^{2}\left(1-\mu^{2}\right)} \\
u_{\mathrm{sh}}=q \sqrt{a_{\mathrm{sh}}^{2} \mu^{2}+b_{\mathrm{sh}}^{2}\left(1-\mu^{2}\right)} \\
V_{\mathrm{c}}=\frac{4}{3} \pi a_{\mathrm{c}} b_{\mathrm{c}}^{2} \\
V_{\mathrm{sh}}=\frac{4}{3} \pi a_{\mathrm{sh}} b_{\mathrm{sh}}^{2}
\end{gathered}
$$

where $\eta_{\mathrm{c}}, \eta_{\mathrm{sh}}$ and $\eta_{\mathrm{m}}$ are respectively the scattering length densities of core, shell and surrounding medium. $j_{1}(u)$ is the first order spherical Bessel function and $\mu$ the orientation variable, equal to the cosine of the angle $\theta$ between the scattering vector $\bar{q}$ and the direction of the spheroid major axis. $I_{\text {part }}(q)$ can then be derived as follows following the integration over $\mu$ :

$$
I_{\text {part }}(q)=<F^{2}(q, \mu)>=\int_{0}^{1} F^{2}(q, \mu) \mathrm{d} \mu
$$

We can then calculate the $q$-dependent intensity along the two principal directions parallel and perpendicular to the stretching direction by setting $\mu$ to 1 or 0 , respectively. Eqn (4) then

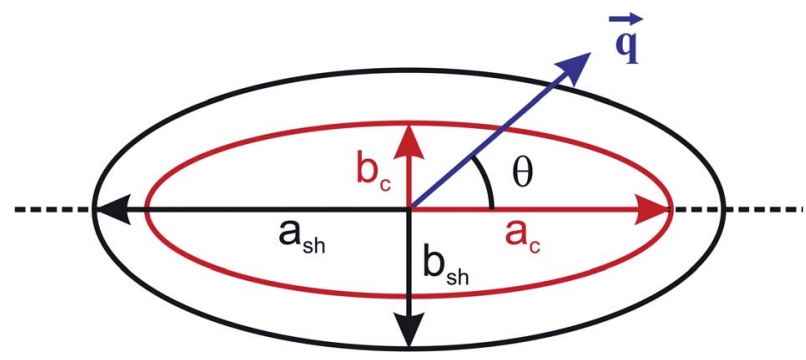

Fig. 4 Schematic model for the core-shell ellipsoid. corresponds to the scattering intensity of polydisperse core-shell spherical particles with core and core-shell radii equal either to $a_{\mathrm{c}}$ and $a_{\mathrm{sh}}$ (short azimuthal) or $b_{\mathrm{c}}$ and $b_{\mathrm{sh}}$ (long azimuthal). For the evaluation of the part of the scattering caused by the static inhomogeneities within the shell $I_{\text {fluc }}(q)$ it is appropriate to use the empirical formula: ${ }^{42,46}$

$$
I_{\text {fluc }}(q)=\frac{I_{\text {fluct }}(0)}{1+\xi^{2} q^{2}}
$$

$I_{\text {fluct }}(0)$ is a constant and the average correlation length in the network is described by $\xi$. $I_{\text {fluc }}$ contributes significantly only in the high $q$ regime.

The scattering intensity profile of the different particles could be fitted, including polydispersity, assuming a Gaussian distribution of the different dimensions. Fig. 3 presents the results obtained for the core and core-shell particles stretched with 0, 50 and $125 \%$ deformation. Here the different $2 \mathrm{D}$ scattering patterns are presented together with the corresponding intensities (azimuthal sector average along the two principal axes and full azimuthal average). A close look at the scattering patterns confirms that the particles were elongated within the films during the stretching. Indeed the unstretched films exhibit an isotropic pattern, whereas the pattern becomes more and more anisotropic with increasing deformation. The data for the films containing the core particles were fitted assuming a polydisperse homogeneous sphere model with an average radius of $267 \mathrm{~nm}$ and a polydispersity of $5 \%$. Hereby we neglect the contribution of the PNIPMAm, which is usually present at the surface of the particles in the form of a thin layer. This model nicely described the experimental data and the characteristic $q^{-4}$ decrease of the scattering intensity at high $q$-values in the Porod regime. Moreover, the resulting size distribution matches the estimates obtained from the TEM. Fig. 3E) presents the same experiments for the core-shell particles. The scattering intensity first decayed with a powerlaw of $q^{-4}$ for intermediate $q$ values, and then with $q^{-2}$ at higher $q$-values characteristic for microgels. The scattering intensities were fitted using a core-shell model, where we also take into account the inhomogeneities within the shell following eqn (5) which mostly contribute at higher $q$. Here we need to consider the electronic contrast of the different elements. We consider an electronic scattering length density $\eta_{\mathrm{c}}=341.2 \mathrm{e} \mathrm{nm}$ for the polystyrene core corresponding to a density $\rho_{\mathrm{PS}}=1.054$ $\mathrm{g} \mathrm{cm}^{-3}$ and $\eta_{\mathrm{m}}=427.0 \mathrm{e} \mathrm{nm}^{-3}$ for the PVA, which corresponds to a density of $\rho_{\text {PVA }}=1.30 \mathrm{~g} \mathrm{~cm}^{-3} \cdot{ }^{54}$ If we fix the size of the core based on the results obtained in the previous experiment, the best fit could be obtained for a shell of $65 \mathrm{~nm}$, assuming a dense shell with a scattering length density of $\eta_{\mathrm{sh}}=364.6 \mathrm{e} \mathrm{nm}^{-3}$ corresponding to a density of $1.10 \mathrm{~g} \mathrm{~cm}^{-3}$ and an overall polydispersity of $7.5 \%$. Our experimental density value is somewhat lower than what could be found in the literature for collapsed $5 \mathrm{~mol} \%$ crosslinked PNIPMAm microgels in the collapsed state $(1.129$ $\mathrm{g} \mathrm{cm}^{-3}$ measured at $\left.55^{\circ} \mathrm{C}\right) .{ }^{55}$ The overall size of $332 \mathrm{~nm}$ derived from this analysis was found to be in good agreement with the overall size of $331 \mathrm{~nm}$ from the TEM. However it remains difficult to determine the exact value experimentally as we do not know the configuration of the PNIPMAm shell in the PVA film.

In order to describe the scattering pattern after stretching, we first assume that the spheres deform affinely in the direction of 
the stretch and that the sphere retains its total mass. As predicted for cold prestretching by Eshelby and Keville et al. ${ }^{28,48}$ the particles should then adopt an ellipsoidal shape, with the long axis scaling $a=1+\gamma$, the short axis $b=(1+\gamma)^{-\frac{1}{2}}$ and an aspect ratio $\rho=a / b=(1+\gamma)^{\frac{3}{2}}$. This also has a direct consequence for the core-shell particles as it implies that the shell in the direction of the short axis $\Delta b_{\mathrm{s}}$ gets thinner, while the shell in the direction of the long axis $\Delta a_{\mathrm{s}}$ should become thicker such that $\Delta a_{\mathrm{s}} / \Delta b_{\mathrm{s}}=\rho$. In our analysis we consider that the total mass is conserved and that the particles adopt an ellipsoidal shape as presented in the insets of the different parts in Fig. 3. The main fit parameters are therefore the aspect ratio and the polydispersity of the particles. The shell density was also slightly vary between 1.08 and 1.1 $\mathrm{g} \mathrm{cm}^{-3}$ during the fitting. We decided not to vary the density of the core. Under these conditions we then fit the different scattering intensities. The polydispersity was increased from 13 to $20 \%$ with increasing $\gamma$. This approach describes our experiments for both core and core-shell particles. The corresponding models are schematically depicted with aspect ratio in Fig. 3. We repeated the procedure for other deformations ranging from 50 to $150 \%$. As summarized in Fig. 5, the different dimensions used for the fitting and the corresponding aspect ratios obtained for the core and the core-shell verify the prediction for a cold prestretch. However, oriented ellipsoidal particles should result in an ellipsoidal pattern in reciprocal space, which is not the case when looking at the SAXS data from the films. Here the patterns, particularly at high deformations, appear more diamond-like than ellipsoidal as a possible consequence of a deviation from an ellipsoidal to a spindle-like shape of the particles.

In order to clarify this issue, the stretched particles were recovered after the dissolution of the films in hot water at $80^{\circ} \mathrm{C}$. They were then purified by repeated centrifugation steps in order to remove the remaining PVA. Based on the mass of the film, the water content was adjusted in order to reach a final concentration close to $1 \mathrm{wt} \%$. The different particles were imaged by TEM in the dried state after dissolution of the PVA film and purification, and examples of the resulting images are shown in Fig. 6 for different draw ratios $\gamma$. Well-defined and nicely dispersed particles could be recovered after the drawing process of the core-shell lattices. Unfortunately the elongated core particles could not be redispersed, most probably as a consequence of the thin layer of PNIPMAm present at their surface. Increasing the draw ratio $\gamma$ leads to an increase of the anisotropy of the particles. At larger $\gamma$, many of the particles deviated from an ellipsoidal shape and instead appear spindle-like. The soft tip of the particles adsorbs on the surface of the grid, which indicates a lower polystyrene content. Assuming an ellipsoidal shape, the long and short axis, as well as the corresponding aspect ratios were evaluated from the TEM micrographs by counting around 100 particles. The results are displayed in Fig. 7 as function of $\gamma$. The error bars correspond to the standard deviation of each measurement. The average long axis was observed to be between 1.94 to $3.61 \mu \mathrm{m}$ and the average short axis from 0.73 to $0.57 \mu \mathrm{m}$ when varying $\gamma$ between 50 and 225\% (Fig. 7(A)). In this deformation range the average aspect ratio could then be adjusted between 2.7 and 6.9 (Fig. 7(B)). The standard deviation for the long axis was found to be around $17 \%$, with a smaller value around $12 \%$ for the short axis almost independent of the applied draw ratio. As a consequence an average standard deviation of $24 \%$ results for the aspect ratio. The particles obtained are then rather polydisperse, particularly because of the lower size control for the long axis. This is larger than what could be obtained for pure polystyrene particles in the work of Ho et al. ${ }^{29}$ where the authors reported a polydispersity below $10 \%$. The lack of control on the elongation speed of our stretching setup and the fact that the films have been stretched in an oven and not in an oil bath may also explain the deviation from the ellipsoidal shape and the larger polydispersity. In Fig. 7, the dashed lines correspond to the prediction for the cold prestretch. Experimentally, it appears that particles undergo a larger deformation than the film. These calculations assumed an isochore deformation and an ellipsoidal shape, while the TEM micrographs clearly demonstrate some deviations from this model. This indicates that the stretched core-shell particles deviate from the ellipsoidal shape and adopt a more elongated "spindle-like" structure in the dried state at high elongations as observed for instance for PMMA particles. ${ }^{30}$

The different systems have been investigated by light scattering in solution before and after the volume phase transition at 20 and
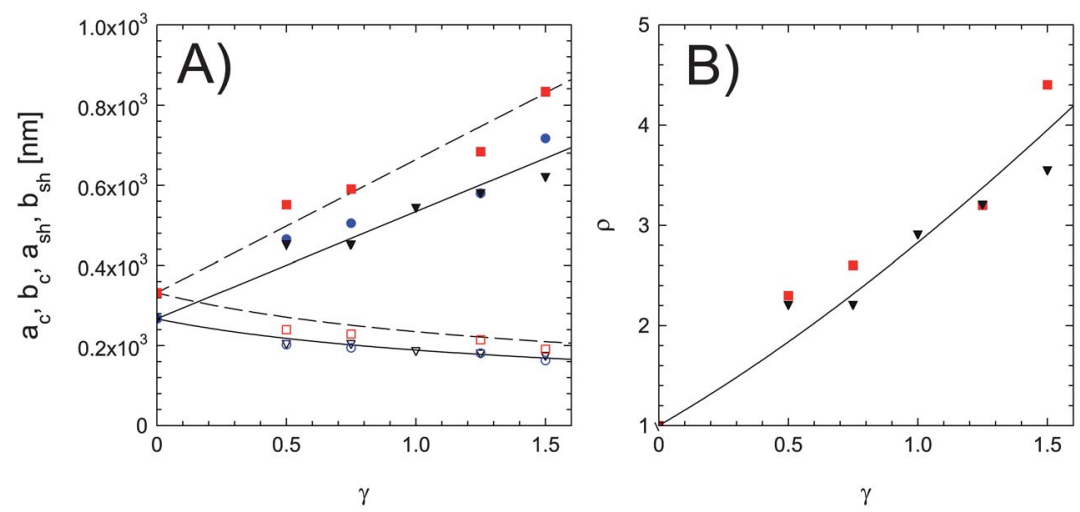

Fig. 5 (A) Evolution of the minor and major semi-axes following the deformation of the films derived from the SAXS analysis of core $\left(a_{\mathrm{c}}\right.$ : full triangles; $b_{\mathrm{c}}$ : hollow triangles) and core-shell ( $a_{\mathrm{c}}$ : full circles; $b_{\mathrm{c}}$ : hollow circles; $a_{\mathrm{sh}}$ : full squares; $b_{\mathrm{sh}}$ : hollow squares) particles. The full and dashed lines present the comparison with the prediction for cold prestretch ${ }^{28,48}$ for the core and core-shell respectively. (B) Corresponding aspect ratios for both particles (core: full triangles; core-shell: full squares) compared with the prediction for an affine isochore deformation (full line). 

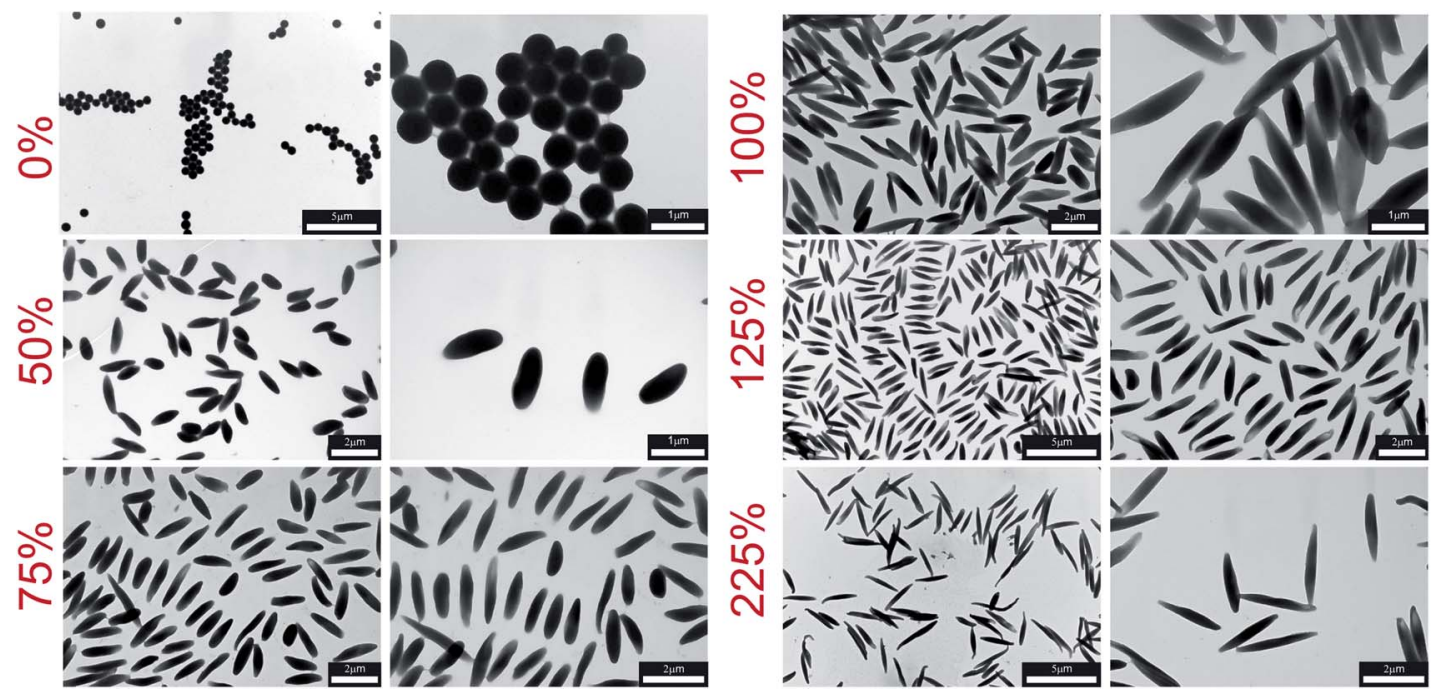

Fig. 6 Electron micrographs of the core-shell particles and of the different ellipsoids obtained at different deformations.

$50{ }^{\circ} \mathrm{C}$ (Fig. 8). The scattering intensity has been recorded for angles between $22^{\circ}$ and $144^{\circ}$, corresponding to a $q$-range of 0.025 to $0.002 \mathrm{~nm}^{-1}$. Due to the small amount of sample it was not possible to determine precisely the initial concentration. Nevertheless, based on the loading of the films, we adjusted the particle concentration to around $10^{-3} \mathrm{wt} \%$ in order to avoid any multiple scattering. The core and core-shell particles as well as the elongated particles obtained after stretching were measured. The data sets obtained at $20{ }^{\circ} \mathrm{C}$ were rescaled so as to have the same forward scattering as the pure core-shell at $20{ }^{\circ} \mathrm{C}$, and then multiplied by the constants indicated in Fig. 8. The same rescaling factor was used for the data measured at $50{ }^{\circ} \mathrm{C}$. As expected for monodisperse spherical particles, the core and coreshell particles exhibit a well-defined first minimum. The dashed lines correspond to the theoretical predictions for homogeneous spheres using the Rayleigh-Gans-Debye (RGD) approximation with the size and polydispersity as determined from the TEM analysis. This approach provides a good qualitative description of the data and of the position of the first maximum, but fails to describe the first minimum. In this size range the scattering is not fully described by RGD, therefore we reiterate the fitting procedure using the Mie model. We calculate the Mie scattering ${ }^{56,57}$ of polydisperse polystyrene particles with a refractive index of 1.59 dispersed in water assuming a Gaussian size distribution. A quantitative description of the experimental data could now be achieved on the full $q$ range using an average size of $240 \mathrm{~nm}$ for the core and $310 \mathrm{~nm}$ for the core-shell particles with polydispersities of 5.6 and $7.5 \%$ respectively.

For the stretched particles, we then calculated the $q$-dependence of the scattering intensity from the average dimensions and polydispersity determined from TEM using RGD and assuming homogeneous ellipsoidal particles. Similarly to the spherical core and core-shell particles, this approach provides a good description of the experimental scattering intensity, except for the smeared out first minimum, which could be again attributed to the Mie scattering of these particles. The discrepancy between the calculated and experimental intensity becomes smaller for the higher aspect ratios. This is not surprising as it is generally the smallest dimension of anisotropic particles that determines whether RGD is still applicable, and with increasing anisotropy and decreasing minor semi-axes the particles then move into the RGD regime. The core-shell and the stretched particles
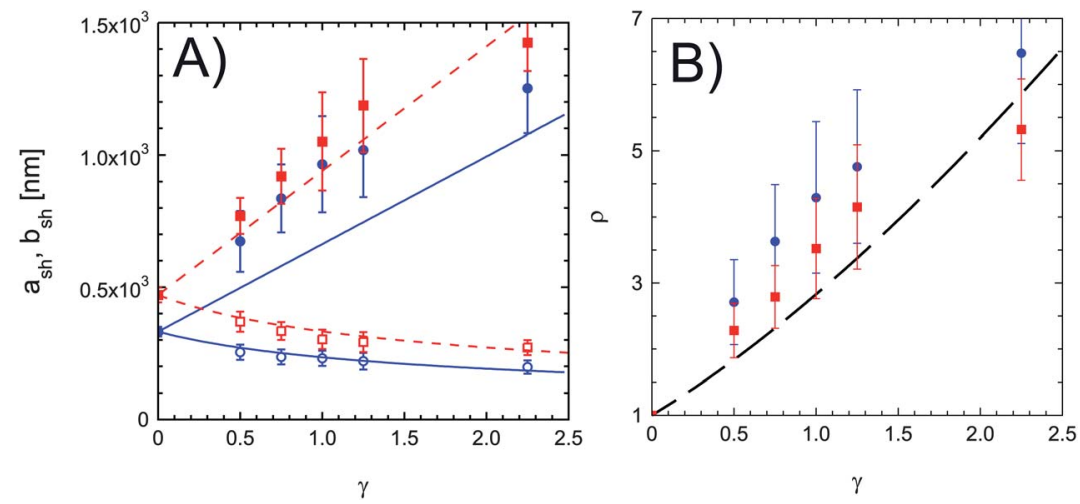

Fig. 7 (A) Evolution of minor (hollow symbols) and major (full symbols) semi-axes $a_{\mathrm{sh}}$ and $b_{\mathrm{sh}}$ as a function of the deformation $\gamma$ determined from the TEM (circles) and CSLM (squares) analysis. The lines present the comparison with the prediction for cold prestretch. (B) $\gamma$-dependence of the aspect ratio $\rho$ derived from TEM (full circles) and CLSM (full squares) in respect of the predictions (dashed line). 


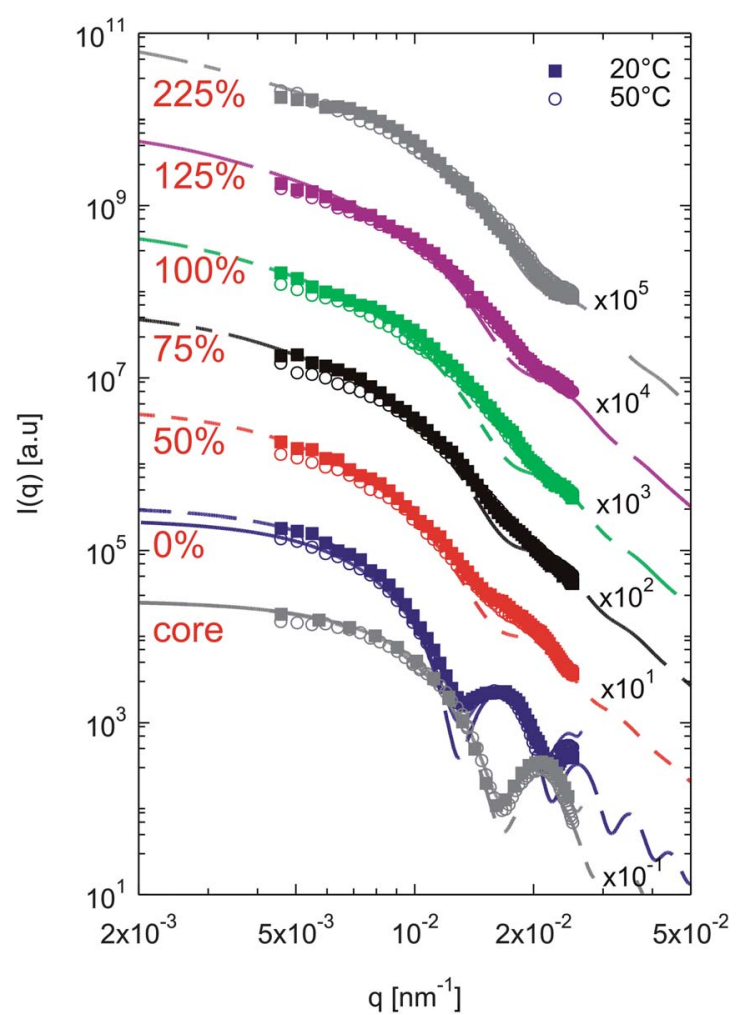

Fig. 8 Static light scattering analysis of the core, core-shell particles and the different ellipsoids obtained for different draw ratios. Full symbols refer to the measurements performed at $20^{\circ} \mathrm{C}$, whereas the hollow ones correspond to the measurement done at $50^{\circ} \mathrm{C}$ in the collapsed state. The dashed lines present the RGD calculation considering the size and polydispersity determined by TEM. As a comparison the full lines are best fittings considering the Mie scattering for the spherical particles (see text for more details).

measured at 20 and $50{ }^{\circ} \mathrm{C}$, before and after the VPT, deviate in the low $q$ region up to $100 \%$ draw ratio. For more elongated particles no significant difference was noticed in this $q$-range.
This is most probably related to the shrinking of the PNIPMAm shell at high temperature. At larger draw ratios, the swelling of the shell is constrained which could explain the reduced effect on the scattering intensity at high temperatures. This will be discussed in more detail from the evaluation of the dynamic light scattering experiments.

The core-shell particles as well as the different ellipsoids could be imaged in suspension by confocal microscopy (Fig. 9). For this purpose we labeled the particles with a positively charged Rhodamine B which could easily be absorbed in the polymeric PNIPMAm shell. The different results obtained for different draw ratios are summarized in Fig. 7. All images were taken at $20{ }^{\circ} \mathrm{C}$ either close to the drying front of the samples or rather close to the surface of the glass where the particles were adsorbed. This allows a good visualization of the particles due to the arrested dynamics. In the middle of the sample the particles could be imaged as well but the fast dynamics did not allow a good resolution of their structure. The spherical particles exhibit a well-defined core-shell structure and appear highly monodisperse, with an average diameter of $470 \pm 28 \mathrm{~nm}$ derived from the confocal microscopy, which is in good agreement with the radius determined by dynamic light scattering $\left(489 \mathrm{~nm}\right.$ at $\left.20^{\circ} \mathrm{C}\right)$. After the stretching step, the particles are now clearly anisotropic, and appear in the form of prolates up to $\gamma=75 \%$ with an aspect ratio that increases with the applied deformation. At smaller $\gamma$ (until $75 \%$ ), the core-shell structure of the particles can still be seen, however at larger $\gamma$ the core of the particles cannot be observed any more. The different dimensions have been estimated from the CLSM micrographs are summarized in Fig. 7 (A). The long axis increases continuously from between 770 and $1424 \mathrm{~nm}$ with increasing deformation, whereas the short axis decreases from 369 to $271 \mathrm{~nm}$, which corresponds to a variation of the aspect ratio between 2.28 and 5.32 (Fig. 7(B)). Both dimensions, as well as the corresponding aspect ratios, could be described by Eshelby's predictions. Moreover this analysis clearly established that the microgels are swelling in solution as the different dimensions are larger than the ones determined by TEM. When comparing the different aspect ratios obtained from
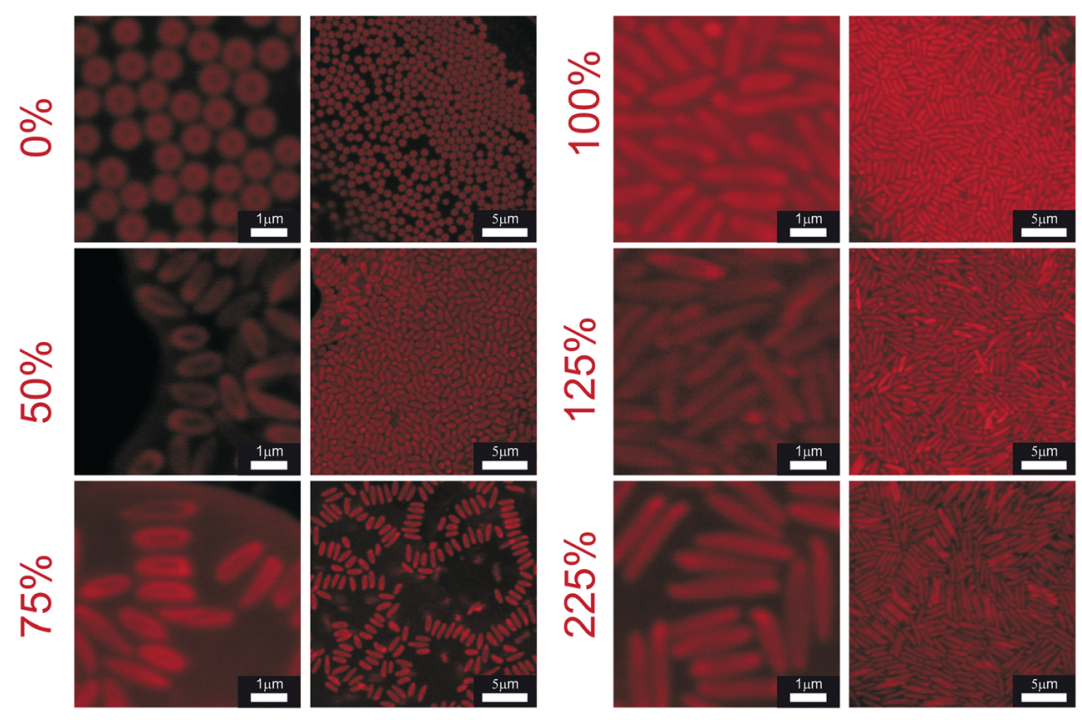

Fig. 9 Confocal micrographs of the core-shell particles and of the elongated core-s-hell particles for the different deformations indicated in the figure. 
the two microscopy experiments, it appears that the CLSM analysis yields lower aspect ratios with respect to TEM. This indicates that not only the dimensions but also the aspect ratio of the particles could be adjusted with temperature.

The volume transition within the shell was studied by DLS. The evolution of the translational diffusion coefficient $D_{\mathrm{T}}$ was determined from the linear dependence of the decay rate $\Gamma$ versus the square of the scattering vector $q^{2}\left(\Gamma=D_{\mathrm{T}} q^{2}\right)$ derived from a first order cumulant analysis. The apparent hydrodynamic radius $R_{\mathrm{H}}$ was determined from $D_{\mathrm{T}}$ via the Stockes-Einstein relation $D=k_{\mathrm{B}} T / 6 \pi \eta R_{\mathrm{H}}$, with $k_{B}$ the Boltzmann constant, $T$ the temperature of the measurement in Kelvin and $\eta$ the viscosity in $\mathrm{Pa}$ s. Fig. 10 shows the evolution of the apparent hydrodynamic radius of the core-shell spherical microgels determined by DLS as a function of the temperature. $R_{\mathrm{H}}$ decreases gradually with temperature from $480 \mathrm{~nm}$ to $457 \mathrm{~nm}$ up to $30{ }^{\circ} \mathrm{C}$ until a sharp volume transition from the swollen to the collapsed state takes place with a transition at around $42{ }^{\circ} \mathrm{C}$. The final radius at a collapsed state of $349 \mathrm{~nm}$ is reached at a temperature above 50 ${ }^{\circ} \mathrm{C}$. The colloidal stability of the different systems was maintained above the VPT because of a residual electrostatic repulsion between the particles due to the small amount of charges on the cores and in the shell. As already discussed, the overall size of the particles was in good agreement with the results from confocal microscopy performed at $20{ }^{\circ} \mathrm{C}$, whereas the value determined in the collapsed state approached the average size in the dried state determined by TEM. This analysis confirmed the thermosensitivity of the different systems. The same procedure was repeated for the different anisotropic particles. Here the DLS analysis shows that the particles maintained their thermosensitivity after the stretching process, with a volume phase transition shifting with increasing aspect ratio from 42 to $38^{\circ} \mathrm{C}$.

At $50 \%$ and $75 \%$ deformation, $R_{\mathrm{H}}$ appears smaller, whereas at higher deformations it increases above the value of the original particles by up to $30 \%$ at $50{ }^{\circ} \mathrm{C}$. Assuming the translational diffusion coefficient of an ellipsoid derived by Perrin ${ }^{58}$ and an isochore deformation, $D_{\mathrm{T}}$ should decrease by $34 \%$ for an aspect ratio of 6.5. This agrees with what we observe for the most anisotropic particles at high temperatures. However it is not the case at low temperatures in the swollen state, where the difference

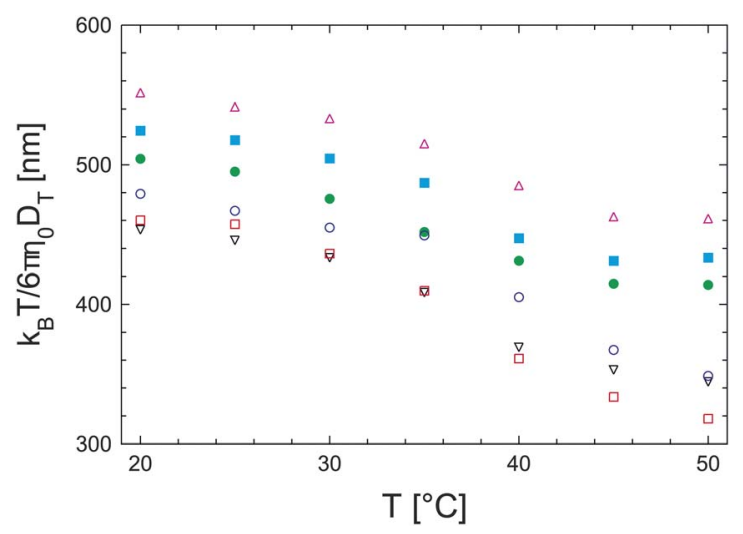

Fig. 10 Temperature dependence of the apparent hydrodynamic radius for the particles stretched at different deformations ( $0 \%$ : hollow circles; $50 \%$ : hollow squares; $75 \%$ : hollow down triangles; $100 \%$ : full circles; $125 \%$ : full squares; and $225 \%$ : hollow up triangles). is much smaller, which also indicates that the stretching process may have reduced the swelling of the particles, similar to that observed in a conventional elastomeric network. The same analogy may also explain the shift of the VPT to lower temperatures on the basis of the entropic elasticity present in loosely crosslinked elastomers. The dependence of the swelling with respect to the deformation of the microgels' shell will be discussed in more detail in another study.

Fig. 11 displays the micrographs obtained by cryo-TEM for the original spherical core-shell particles at room temperature and for the particles elongated with $75 \%$ deformation at two different temperatures following the method described previously. ${ }^{43}$ We first consider the micrographs related to room temperature (Fig. 5A). In order to enhance the contrast a background subtraction, consisting of a low and high pass filter, was performed on the micrographs. The spherical particles appeared narrowly distributed. Moreover the PNIPMAm shell was clearly visible even without requiring any staining agent covering every single particle. When compared to a former analysis of smaller PS/PNIPMAm particles, ${ }^{39,43,44}$ the shell does not exhibit inhomogeneities and appears uniformly attached to the core of the particles, whereas the smaller particles investigated earlier clearly showed some buckling at the core-shell interface. Due to their size in the micrometre range, the films were much thicker than in the former analysis. Therefore, multiple electron scattering occurred, explaining the lack of contrast observed in the micrographs. This is illustrated by the comparison with the hydrodynamic radius determined in solution at $25{ }^{\circ} \mathrm{C}$ by DLS, much

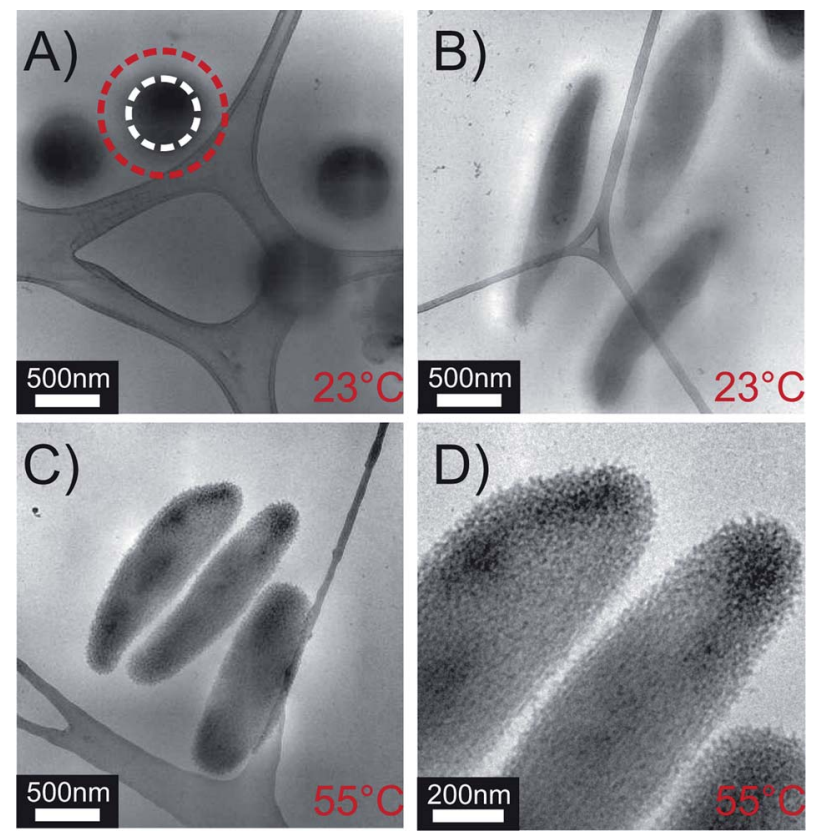

Fig. 11 Cryo-TEM micrographs of a $0.2 \mathrm{wt} \%$ aqueous suspensions of PS/PNIPMAm spherical core-shell particles maintained at room temperature before vitrification (A). The two dashed circles indicate the core and core-shell radii determined by dynamic light scattering at $20^{\circ} \mathrm{C}$. The same experiment was repeated for the particles obtained at a draw ratio of $75 \%$, where the particles are equilibrated at room temperature (B) and at $55^{\circ} \mathrm{C}$, i.e. above the VPT for the cross-linked PNIPMAm shell (C and D). 
larger than the overall size of the particles (see dashed circle in Fig. 11). The same procedure was repeated in the case of the elongated particles quenched from room temperature. The shell appeared fuzzy as could be observed on the spherical particles. The cores, clearly distinguishable from the different micrographs, exhibit an inhomogeneous deformation. Moreover the shells appear to be thicker at the edges, consistent with the confocal and TEM microscopy images. The picture changed completely when the particles were quenched from $55^{\circ} \mathrm{C}$. The collapse of the shell is clearly visible (Fig. 11C and D). The shell becomes denser and has a higher contrast, therefore it becomes difficult to distinguish its interface with the core. This suggests different conformations in the structure of the PNIPMAm shell, and most probably indicates at two different relaxation processes. On the long axis of the thus obtained ellipsoidal particles, the shell systematically appeared as being highly elongated after the deformation of the core, whereas on the edge of the particles, the shell could undergo a more homogeneous deswelling. Whereas many experiments have focused on the structure and swelling of stretched macrogels, particularly with scattering techniques, these experiments show for the first time the direct visualization of the VPT of constrained microgels at the nanoscale.

\section{Conclusion}

Anisotropic ellipsoidal-shaped functional particles were obtained by mechanical stretching of composite core-shell microgels. The aspect ratio of these particles can be varied almost at will. While the stretching conditions determine the aspect ratio of the core, the overall aspect ratio of the composite particles can then be further tuned through the thermoresponsive shell. This allows the control of the effective volume fraction with temperature, related to the swelling of the shell, but also the potential of the particles. This new class of microgels thus represents perfect candidates for future investigation of the phase diagram of ellipsoidal particles, for which the preparation of the sample at high volume fraction is a major issue. Such problems can be easily overcome with microgels, as the history of the sample can be simply erased by a fast temperature quench from the liquid disordered state to higher volume fractions. Moreover the control of the interactions combined with the anisotropy of the particles open the door to more complex phase diagrams, closer to biological systems where morphological and interactive anisotropy play a major role.

\section{Acknowledgements}

We acknowledge financial support by the Adolphe Merkle Foundation and the Swiss National Foundation. We would like to particularly thank Hans-Peter Kuenzi for the dynamic light scattering measurements.

\section{References}

1 S. C. Glotzer and M. J. Solomon, Nat. Mater., 2007, 6, 557.

2 M. J. Solomon and P. T. Spicer, Soft Matter, 2010, 6, 1391.

3 S. Sacanna and D. J. Pine, Curr. Opin. Colloid Interface Sci., 2011, 16, 96-105.

4 M. J. Solomon, Curr. Opin. Colloid Interface Sci., 2011, 16, 158.
5 S. N. Fejer, D. Chakrabartia and D. J. Wales, Soft Matter, 2011, 7, 3553.

6 Y. Han, A. M. Alsayed, M. Nobili, J. Zhang, T. C. Lubensky and A. G. Yodh, Science, 2006, 314, 626-630.

7 D. Mukhija and M. J. Solomon, J. Colloid Interface Sci., 2007, 314, 98.

8 I. Martchenko, H. Dietsch, C. Moitzi and P. Schurtenberger, J. Phys. Chem. B, 2011, 115, 14838.

9 A. Donev, F. H. Stillinger, P. M. Chaikin and S. Torquato, Phys. Rev. Lett., 2004, 92, 255506.

10 A. Donev, I. Cisse, D. Sachs, E. Variano, F. H. Stillinger, R. Connelly, S. Torquato and P. M. Chaikin, Science, 2004, 303, 990.

11 P. Pfleiderer and T. Schilling, Phys. Rev. E: Stat., Nonlinear, Soft Matter Phys., 2007, 75, 020402.

12 P. Pfleiderer, K. Milinkovic and T. Schilling, Europhys. Lett., 2008, 84, 16003.

13 M. Radu, P. Pfleiderer and T. Schilling, J. Chem. Phys., 2009, 131, 164513.

14 H. Maeda and Y. Maeda, Phys. Rev. Lett., 2003, 90, 18303.

15 P. P. Lele and E. M. Furst, Langmuir, 2009, 25, 8875.

16 Y. Lu, Y. D. Yin, Z. Y. Li and Y. N. Xia, Langmuir, 2002, 18, 7722.

17 A. Sanchez-Ferrer, R. Mezzenga and H. Dietsch, Macromol. Chem. Phys., 2011, 212, 627-634.

18 E. Matijevic and P. Schreiner, J. Colloid Interface Sci., 1978, 63, 509.

19 C. Pacholski, A. Kornowski and H. Weller, Angew. Chem., Int. Ed., 2002, 41, 1188.

20 M. Ocana, M. P. Morales and C. J. Serna, J. Colloid Interface Sci., 1999, 212, 317-323.

21 H. Dietsch, V. Malik, M. Reufer, C. Dagallier, A. Shalkevich, M. Saric, T. Gibaud, F. Cardinaux, F. Scheffold, A. Stradner and P. Schurtenberger, Chimia, 2008, 62, 805-814.

22 M. Reufer, H. Dietsch, U. Gasser, B. Grobety, A. M. Hirt, V. K. Malik and P. Schurtenberger, J. Phys.: Condens. Matter, 2011, 23, 065102.

23 S. Sacanna, L. Rossi, B. W. M. Kuipers and A. P. Philipse, Langmuir, 2006, 22, 1822-1827.

24 M. Reufer, H. Dietsch, U. Gasser, A. Hirt, A. Menzel and P. Schurtenberger, J. Phys. Chem. B, 2010, 114, 4763.

25 C. Rufier, M. Reufer, H. Dietsch and P. Schurtenberger, Langmuir, 2011, 27, 6622-6627.

26 C. Dagallier, H. Dietsch, P. Schurtenberger and F. Scheffold, Soft Matter, 2010, 6, 2174.

27 A. Sanchez-Ferrer, M. Reufer, R. Mezzenga, P. Schurtenberger and H. Dietsch, Nanotechnology, 2010, 21, 185603.

28 K. M. Keville, E. I. Franses and J. M. Caruthers, J. Colloid Interface Sci., 1991, 144, 103.

29 C. C. Ho, A. Keller, J. A. Odell and R. H. Ottewill, Colloid Polym. Sci., 1993, 271, 469

30 A. Mohraz and M. J. Solomon, Langmuir, 2005, 21, 5298.

31 Z. K. Zhang, P. Pfleiderer, A. B. Schofield, C. Clasen and J. Vermant, J. Am. Chem. Soc., 2011, 133, 392.

32 J. A. Champion, Y. K. Katare and S. Mitragotri, Proc. Natl. Acad. Sci. U. S. A., 2007, 104, 11901.

33 M. Basavaraj, J. Fransaer and J. Vermant, Langmuir, 2009, 25, 2718.

34 B. Madivala, S. Vandebril, J. Fransaer and J. Vermant, Soft Matter, 2009, 5, 1717

35 J. P. Singh, P. P. Lele, F. Nettesheim, N. J. Wagner and E. M. Furst, Phys. Rev. E: Stat., Nonlinear, Soft Matter Phys., 2009, 79, 050401.

36 M. Mittal and E. M. Furst, Adv. Funct. Mater., 2009, 19, 3271.

37 D. Mukhija and M. J. Solomon, Soft Matter, 2011, 7, 540.

38 X. Guo and M. Ballauff, Macromolecules, 1999, 32, 6043.

39 J. J. Crassous, A. Wittemann, M. Siebenbuerger, M. Schrinner, M. Drechsler and M. Ballauff, Colloid Polym. Sci., 2008, 286, 805.

40 J. J. Crassous, M. Siebenbuerger, M. Ballauff, M. Drechsler, O. Henrich and M. Fuchs, J. Chem. Phys., 2006, 125, 204906.

41 J. J. Crassous, M. Siebenbuerger, M. Ballauff, M. Drechsler, D. Hajnal, O. Henrich and M. Fuchs, J. Chem. Phys., 2008, 128, 204902.

42 S. Seelenmeyer, I. Deike, S. Rosenfeldt, C. Norhausen, N. Dingenouts, M. Ballauff and T. Narayanan, J. Chem. Phys., 2001, 114, 10471.

43 J. J. Crassous, M. Ballauff, M. Drechsler, J. Schmidt and Y. Talmon, Langmuir, 2006, 22, 2403. 
44 J. J. Crassous, C. N. Rochette, A. Wittemann, M. Schrinner and M. Ballauff, Langmuir, 2009, 25, 7862.

45 A. Zaccone, J. J. Crassous, B. Béri and M. Ballauff, Phys. Rev. Lett., 2011, 107, 168303

46 N. Dingenouts, S. Seelenmeyer, I. Deike, S. Rosenfeldt, M. Ballauff, P. Lindner and T. Narayanan, Phys. Chem. Chem. Phys., 2001, 3, 1169.

47 J. Brandrup and E. H. Immergut, Polymer Handbook, 3rd ed., Wiley, New York, 1989.

48 J. D. Eshelby, Proc. R. Soc. London, Ser. A, 1957, 241, 376-396.

49 I. Berndt, J. S. Pedersen and W. Richtering, J. Am. Chem. Soc., 2005, 127, 9372.

50 N. Dingenouts, C. Norhausen and M. Ballauff, Macromolecules, 1998, 31, 8912.
51 N. Dingenouts, J. Bolze, D. Pötschke and M. Ballauff, Adv. Polym. Sci., 1999, 144, 1.

52 C. Behler, J. Caruthers and E. Franses, J. Chem. Phys., 1990, 92, 140 .

53 C. Behler, J. Caruthers and E. J. Franses, J. Chem. Phys., 1993, 98, 3600.

54 K. Nakanishi and M. Kurata, Bull. Chem. Soc. Jpn., 1960, 33, 152157.

55 I. Berndt and W. Richtering, Macromolecules, 2003, 36, 8780.

56 We employed the program Mieplot developed by Philip Laven, which can be downloaded at no charge from http://www.philiplaven.com/ mieplot.htm.

57 P. Laven, Appl. Opt., 2003, 42, 436.

58 F. Perrin, J. Phys. Radium, 1936, 7, 1. 